\title{
Performance Analysis of a Six-Port Receiver in a WCDMA Communication System including a Multipath Fading Channel
}

\author{
A. O. Olopade and M. Helaoui \\ Intelligent RF Radio Technology Laboratory (iRadio Lab), Department of Electrical and Computer Engineering, \\ Schulich School of Engineering, University of Calgary, Calgary, AB, Canada T2N 1N4
}

Correspondence should be addressed to A. O. Olopade; aoolopad@ucalgary.ca

Received 4 October 2013; Accepted 20 November 2013; Published 23 January 2014

Academic Editor: Alexander Koelpin

Copyright (C) 2014 A. O. Olopade and M. Helaoui. This is an open access article distributed under the Creative Commons Attribution License, which permits unrestricted use, distribution, and reproduction in any medium, provided the original work is properly cited.

\begin{abstract}
Third generation communication systems require receivers with wide bandwidth of operation to support high transmission rates and are also reconfigurable to support various communication standards with different frequency bands. An ideal software defined radio (SDR) will be the absolute answer to this requirement but it is not achievable with the current level of technology. This paper proposes the use of a six-port receiver (SPR) front-end (FE) in a WCDMA communication system. A WCDMA end-to-end physical layer MATLAB demo which includes a multipath channel distortion block is used to determine the viability of the six-port based receiver. The WCDMA signal after passing through a multipath channel is received using a constructed SPR FE. The baseband signal is then calibrated and corrected in MATLAB. The six-port receiver performance is measured in terms of bit error rate (BER). The signal-to-noise ratio (SNR) of the transmitted IQ data is varied and the BER profile of the communication system is plotted. The effect of the multipath fading on the receiver performance and the accuracy of the calibration algorithm are obtained by comparing two different measured BER curves for different calibration techniques to the simulated BER curve of an ideal receiver.
\end{abstract}

\section{Introduction}

Third generation $(3 \mathrm{G})$ mobile communication systems introduced in recent years are a huge step in increasing wireless transmission capacity, fidelity, and efficiency. The increasing number of cellular standards together with the variety of frequency bands these standards use in different regions of the world demands a high degree of reconfigurability. The idea of reconfigurability applies not only to the baseband processing but also to the RF front-end. As a result, reconfiguration has become the key issue in the design of wireless terminals [1]. The implication is that the receiver front-end (FE) is required to have a wide bandwidth to support a high data transmission rate and it should also be multimode and multistandard to support fast and constantly evolving modern communication systems. This enables forward and backward compatibility. Power requirement, fidelity, size, and cost are also paramount properties to consider in a receiver front-end design.

An example of $3 \mathrm{G}$ mobiles communication standard is the WCDMA communication systems, which compared to the second generation systems have a larger system capacity and greater coverage area to provide higher transmission rate and more services to consumers.

An ideal software defined radio (SDR) will be the optimum solution to satisfy the listed requirements on the receiver FE. The SDR radio directly digitizes the output from an antenna and all the receiver blocks (filters, amplifiers, frequency down converters, power detectors, etc.) are implemented in the application software or the embedded systems. Figure 1 describes the structure of an ideal SDR.

However, due to the required high sampling frequency and power dissipation of the analog-to-digital converter (ADC), the ideal SDR has not been feasible with the current technology. Receiver FEs such as direct conversion receivers (DCR) and subsampling receivers have been close candidates to an ideal SDR but however have their peculiar challenges. The DCR is able to do away with the surface acoustic wave (SAW) filter which is typical of superheterodyne receivers, thus reducing size, cost, and complexity. This also enables an integrated single chip solution. However, design of such 


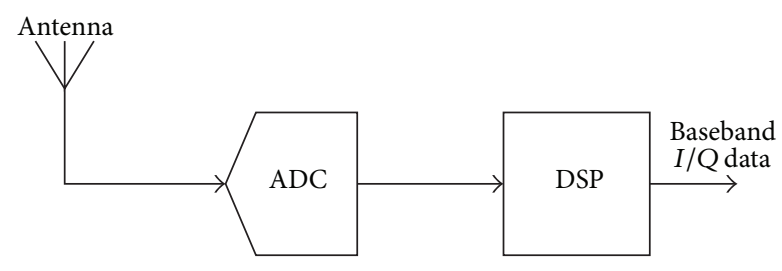

Figure 1: Ideal Software Defined Radio Configuration.

low-cost SAW-less single chip multiband receivers poses significant challenges. The required thresholds on the noise figure $(\mathrm{NF})$, second-order intermodulation intercept point (IIP2), third-order intermodulation intercept point (IIP3), and local oscillator (LO) phase noise are quite challenging to attain. Also, the voltage-control oscillator (VCO) frequency tuning range, and analog-to-digital converter (ADC) signalto-noise ratio (SNR) and dynamic-range requirements to enable a multiband and multimode operation are quite challenging to achieve [2]. DC offset, $I / Q$ mismatch, and LO leakage are some of the drawbacks when these requirements are not met. The subsampling receiver on the other hand has a highly reconfigurable structure because bulk of the demodulation process is done in the digital domain. It however has limitation on bandwidth. Typical design challenges associated with the RF subsampling receiver include the track and hold thermal noise folding effects, aliasing and aperture jitter noise generated from the sampling clock phase noise [3].

Perez-Duenas et al. [4] proposed a six-port based rake receiver for direct sequence ultrawideband systems (DSUWB), which has been considered a type of physical layer (PHY) suitable for high-speed wireless personal area networks (WPANs) in IEEE 802.15.3a. The six-port receiver (SPR) is an alternative direct conversion receiver whose structure is composed of a passive six-port wave correlator and four diode power detectors. While the SPR is typically broadband and highly reconfigurable, its performance is degraded due to the nonidealities in the wave correlator circuit and the limited dynamic range of the diode power detectors. This necessitates driving the detectors outside of their square law region and a suitable calibration technique to mitigate these structural drawbacks to achieve good performance across a broad frequency band at the cost of increasing the computation complexity. The SPR is even more appealing in concurrent dual band receiver operation which uses the same architecture as in the single band case, thus avoiding hardware component duplication which is typical in state-of-the-art concurrent dual band receivers [5]. While previous work investigated some SPR configurations requiring low power consumption and no calibration for low power applications such as millimeter-wave (especially in the $60 \mathrm{GHz}$ band), this work proposes the use of a six-port receiver (SPR) FE for a WCDMA communication system. The SPR is implemented as the receiver FE and the bit error rate of the received signal is used as an index to measure the fidelity of the communication system. The remainder of this paper is structured as follows. Section 2 introduces the six-port receiver theory and discusses its implementation in the WCDMA communication system. Section 3 describes the measurement setup. Section 4 details the results of the receiver and Section 5 concludes the paper.

\section{Six-Port Receiver (SPR) Technique}

2.1. Six-Port Receiver Theory. Among the first use of the sixport technique for a receiver application is the work of $\mathrm{Li}$ et al. in [6]. Due to its simplicity and broad bandwidth, a lot of work has been done to improve its performance and extend its usage [4-12]. A six-port receiver comprises of a six-port wave correlator and four envelope power detectors. As shown in Figure 2, the wave correlator is configured using three quadrature hybrid couplers and a power divider. Diode power detectors (D3 to D6) are typically used as the envelope detectors of the output at ports P3 to P6. The LO signal and the RF have the same center frequency for homodyne operation and are fed through ports $\mathrm{P} 1$ and $\mathrm{P} 2$, respectively. The SPR operates using an addictive mixing of the RF and LO signal in the SPR wave correlator and then a square law processing by the diode power detectors. The wave correlator has predefined phase-shifts and attenuations for the LO and the RF signals such that these two input signals generate different amplitudes and phases at the four output ports [7].

The IQ information can be recovered from the four diode measurement at the output ports. Equation (1) gives the diode output power $\left(P_{i}\right)$ expression in terms of the $S$-parameters of the six-port wave correlator as follows:

$$
P_{i}=\left|S_{i 1}\left(a_{\mathrm{LO}}\right)+S_{i 2}\left(a_{\mathrm{RF}}\right)\right|^{2} \quad \text { for } i=3,4, \ldots 6,
$$

where $a_{\mathrm{LO}}=(1 / \sqrt{2})\left|a_{\mathrm{LO}}\right| e^{j\left(\omega t+\emptyset_{\mathrm{LO}}\right)}$ is the LO signal and $a_{\mathrm{RF}}=(1 / \sqrt{2})\left|a_{\mathrm{RF}}\right| e^{j\left(\omega t+\emptyset_{\mathrm{RF}}\right)}$ is the RF signal.

Expanding (1), the difference equation between two diode outputs is given by

$$
\begin{aligned}
P_{i}-P_{j}= & \left(\left|S_{i 1}\right|^{2}-\left|S_{j 1}\right|^{2}\right)\left|a_{\mathrm{LO}}\right|^{2} \\
& +\left(\left|S_{i 2}\right|^{2}-\left|S_{j 2}\right|^{2}\right)\left(I_{\mathrm{RF}}^{2}-Q_{\mathrm{RF}}^{2}\right) \\
& +2 I_{\mathrm{RF}}\left|a_{\mathrm{LO}}\right|\left\{\left|S_{i 1}\right|\left|S_{i 2}\right| \cos \left(\angle S_{i 2}-\angle S_{i 1}-\emptyset_{\mathrm{LO}}\right)\right. \\
& \left.-\cos \left(\angle S_{j 2}-\angle S_{j 1}-\emptyset_{\mathrm{LO}}\right)\right\} \\
& -2 Q_{\mathrm{RF}}\left|a_{\mathrm{LO}}\right|\left\{\left|S_{i 1}\right|\left|S_{i 2}\right| \sin \left(\angle S_{i 2}-\angle S_{i 1}-\emptyset_{\mathrm{LO}}\right)\right. \\
& \left.-\sin \left(\angle S_{j 2}-\angle S_{j 1}-\emptyset_{\mathrm{LO}}\right)\right\},
\end{aligned}
$$

As mentioned earlier, the ideal six-port wave correlator has specific predefined phase and amplitude relationship for optimum performance in the demodulation process. These conditions must be constant and valid over the entire bandwidth of operation of the SPR [8]. 


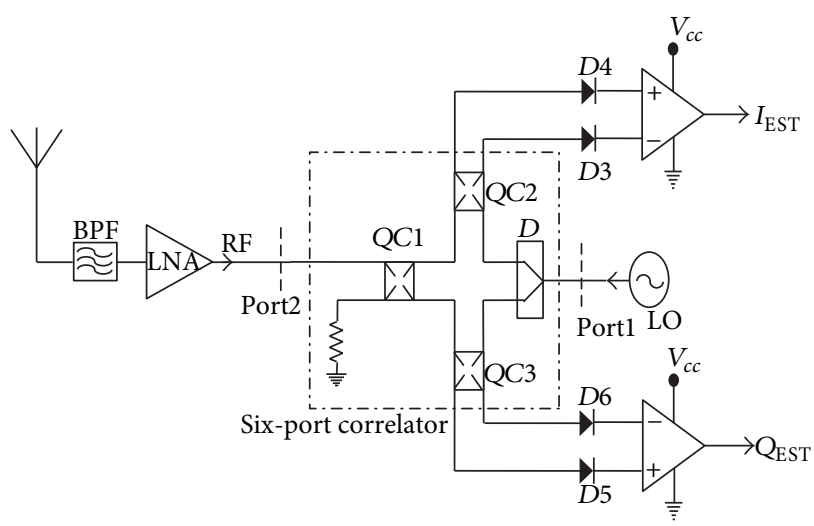

(a)

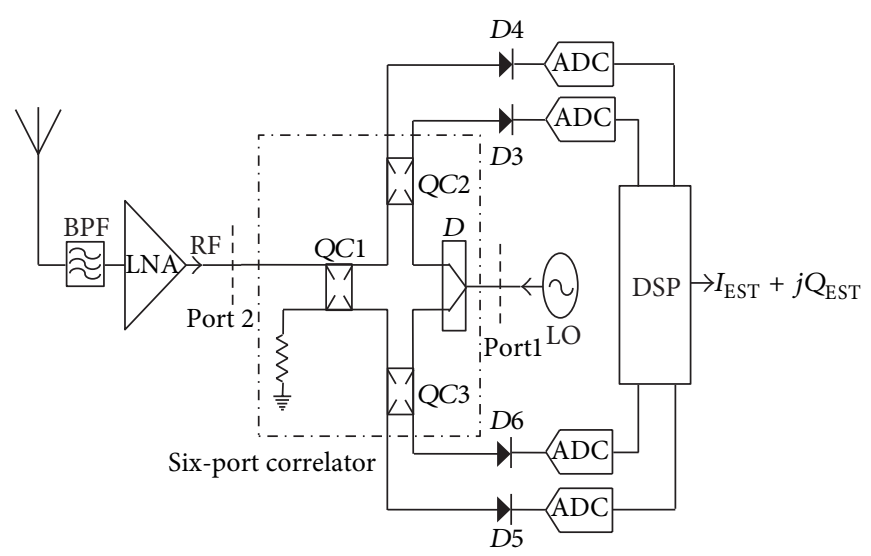

(b)

FIGURE 2: (a) SPR architecture using quasi-ideal component. (b) SPR architecture with DSP estimation.

(i) The conditions on the wave correlator are as follows:
(1) $\left|S_{41}\right|=\left|S_{31}\right|$ and $\left|S_{51}\right|=\left|S_{61}\right|$,
(2) $\left|S_{42}\right|=\left|S_{32}\right|$ and $\left|S_{52}\right|=\left|S_{62}\right|$,
(3) $\angle S_{41}=\angle S_{31}+90^{\circ}$ and $\angle S_{32}=\angle S_{42}+90^{\circ}$,
(4) $\angle S_{51}=\angle S_{61}+90^{\circ}$ and $\angle S_{62}=\angle S_{52}+90^{\circ}$,
(5) $\angle S_{42}-\angle S_{31}-\emptyset_{\mathrm{LO}}=2 n \pi, n=0,1,2 \ldots$,
(6) $\angle S_{52}-\angle S_{61}-\emptyset_{\mathrm{LO}}=(2 n+1 / 2) \pi, n=0,1,2 \ldots$,

where $S_{i j}$ are the $S$-parameters of the wave correlator and $\emptyset_{\mathrm{LO}}$ is the initial phase of the LO signal.

In addition, all four diode detectors must operate within their square law region $\left(P_{i}=K_{i} V_{i}, i=3,4 \cdots 6\right)$ at all times and they must have identical response; that is, $K_{i}$ is the same for $i=3,4, \cdots 6$.

Considering Figure 2(a) which depicts an SPR using quasi-ideal component, the In-phase $(I)$ component of the RF signal is proportional to the difference between diode voltage outputs $V_{3}$ and $V_{4}$ while the quadrature $(Q)$ component is proportional to the difference between $V_{5}$ and $V_{6}$. Equations ( $3 a$ ) and ( $3 b$ ) below give the expression to estimate the $I$ and $Q$ data using architecture the following:

$$
\begin{gathered}
I_{\mathrm{EST}}=\frac{B_{I} K_{2}\left(V_{4}-V_{3}\right)}{4\left|S_{31}\right|\left|S_{32}\right|\left|a_{\mathrm{LO}}\right|}, \\
Q_{\mathrm{EST}}=\frac{B_{\mathrm{Q}} K_{1}\left(V_{5}-V_{6}\right)}{4\left|S_{51}\right|\left|S_{52}\right|\left|a_{\mathrm{LO}}\right|} ;
\end{gathered}
$$

$B_{I}$ and $B_{Q}$ are the gains of the differential amplifiers in the $I$ and $Q$ recovery paths, respectively. Using difference amplifiers as depicted in Figure 2(a), the $I$ and $Q$ data are received directly. However, the assumption of the outlined ideal condition is only valid at the design frequency. This restricts the SPR to a narrow band receiver as a shift from the optimum conditions will result in DC offset and $I / Q$ mismatch thus reducing the fidelity of the receiver. Figure 2(b) shows an architecture which digitizes the output of the diode detectors and uses suitable calibration technique to estimate the $I / Q$ data. A lot of research has been done in SPR calibration using both analog and digital techniques $[6-8,13-$ $15]$, with varying degree of complexity and performance. One such calibration technique is a linear combination of all four diode outputs for the $I / Q$ data estimation as given in the following expressions:

$$
\begin{gathered}
I_{\mathrm{EST}}=\sum_{i=3}^{6} \alpha_{i} P_{i}, \\
\mathrm{Q}_{\mathrm{EST}}=\sum_{i=3}^{6} \beta_{i} P_{i} .
\end{gathered}
$$

Hasan and Helaoui in [8] proposed a modified memory polynomial modeling technique for the SPR which simultaneously compensates for the six-port wave correlator and diode detectors nonidealities. The following equation is the proposed $I / Q$ estimation algorithm:

$$
(I+j Q)_{\mathrm{EST}}(n)=\sum_{d=3}^{6} \sum_{q=0}^{M} \sum_{p=1}^{N} A_{p q d} v_{d}^{p}[n-q],
$$

where $A_{p q d}$ are the complex predetermined calibration constants from a training signal. $M$ is the memory depth and $N$ is the nonlinearity order of the modified memory polynomial for the $n$th symbol. The error vector magnitude (EVM) of the received signal as compared to the transmitted signal was used as the metric to measure the performance of the calibration technique. This calibration technique was reported to have a better performance than the conventional linear calibration techniques.

2.2. Six-Port Receiver in a WCDMA System. Figure 3 shows a MATLAB demo of the WCDMA end-to-end physical layer. It simulates the downlink (DL) path of the frequency division duplex (FDD) downlink physical layer of the WCDMA wireless communication system with the inclusion of the receiver FE which is the focal point of this work. The model has 8 main subsystems listed in Table 1 below with a brief description of their functions. 


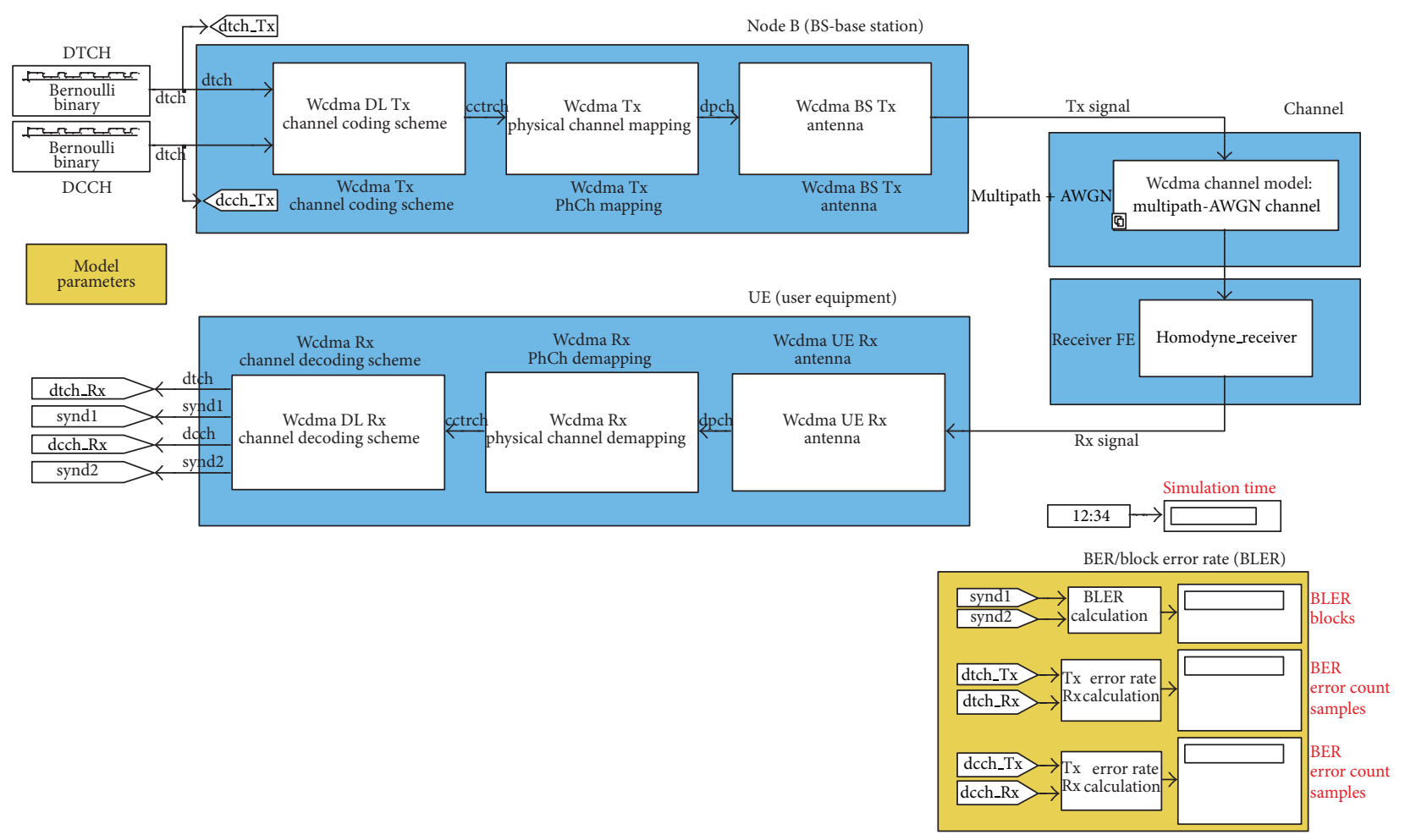

Figure 3: MATLAB Simulink WCDMA end-to-end physical layer demo.

TABLE 1: WCDMA end-to-end physical layer subsystems.

\begin{tabular}{lll}
\hline & Subsystem & Function \\
\hline 1 & WCDMA DL Tx channel coding scheme & Transport channel encoding and multiplexing \\
3 & WCDMA Tx physical mapping & Physical channel mapping \\
4 & WCDMA BS Tx antenna spreading and modulation & Modulation and spreading \\
5 & WCDMA channel model & Channel model \\
6 & Receiver front-end & RF signal reception and frequency demodulation \\
7 & WCDMA UE Rx antenna & Despreading and demodulation \\
8 & WCDMA RX physical channel decoding scheme & Physical channel demapping \\
\hline
\end{tabular}

Detailed description of the subsystems can be found in [16]. A constructed SPR is used as the receiver FE as depicted in the model. A detailed description of the setup for the SPR is given in Section 4.

The DL channels of the demo were set to $384 \mathrm{kbps}$ with the default transport block size of [3840 100]. The number of filter taps for the root raised cosine filter was set to 96 and the number of coefficient for the channel estimation filters was 21 . The oversampling factor was set to 8 . The channel is a multipath channel block with four multipath channels and an additive white Gaussian noise (AWGN) source block. The multipath delay channels were set with relative delays of 0 secs, $260 e-9$ secs, $521 e-9$ secs, and $781 e-9$ secs. The corresponding vector of the average power for the delay paths is $[0,-3,-6,-9] \mathrm{dB}$. The speed of the terminal to model the Doppler Effect was set to $120 \mathrm{Kmph}$. The signal-to-noise ratio of the AWGN was varied between $-15 \mathrm{~dB}$ and $5 \mathrm{~dB}$ in steps of $2.5 \mathrm{~dB}$ to plot the BER profile of the receiver system.

\section{SPR FE Implementation}

The SPR test bench is assembled as in Figure 4. The sixport wave correlator is constructed using three quadrature hybrid couplers Q1 to Q3 and a Wilkinson combiner. Four 8472B Schottky detectors from Agilent Technologies Inc. were used as the power detectors. The generated baseband IQ data at the output of the channel subsystem of the MATLAB Simulink model is up-converted to $2.5 \mathrm{GHz} \mathrm{RF}$ using a signal generator (E4438C ESG from Agilent Inc.). The LO signal also at $2.5 \mathrm{GHz}$ is generated using a second signal generator (E8247C from Agilent Technologies). The RF and LO signals 
TABLE 2: Estimated EVMs of receiver FE at all SNRs.

\begin{tabular}{|c|c|c|c|c|}
\hline $\mathrm{SNR}(\mathrm{dB})$ & $\begin{array}{c}\text { Matlab_wo_multipath } \\
\text { BER (\%) }\end{array}$ & $\begin{array}{c}\text { Matlab_w_multipath } \\
\text { BER (\%) }\end{array}$ & $\begin{array}{c}\text { SPRmp_w_multipath } \\
(N=2, M=4) \\
\text { BER }(\%)\end{array}$ & $\begin{array}{c}\text { SPRc_w_multipath } \\
(N=1, M=0) \\
\text { BER }(\%)\end{array}$ \\
\hline-15 & 0.47180 & 0.4697 & 0.4687 & 0.4707 \\
\hline-12.5 & 0.47000 & 0.4695 & 0.4686 & 0.4701 \\
\hline-10 & 0.46910 & 0.4633 & 0.4636 & 0.4625 \\
\hline-7.5 & 0.45900 & 0.4122 & 0.4135 & 0.4167 \\
\hline-5 & 0.30040 & 0.2301 & 0.2346 & 0.2357 \\
\hline-2.5 & 0.022310 & 0.04113 & 0.04258 & 0.04486 \\
\hline 0 & $1.53 e-05$ & 0.00154 & 0.00165 & 0.001945 \\
\hline 2.5 & 0 & $1.021 e-5$ & $3.064 e-5$ & $4.085 e-5$ \\
\hline 5 & 0 & $5.106 e-6$ & $5.106 e-6$ & $5.106 e-6$ \\
\hline
\end{tabular}

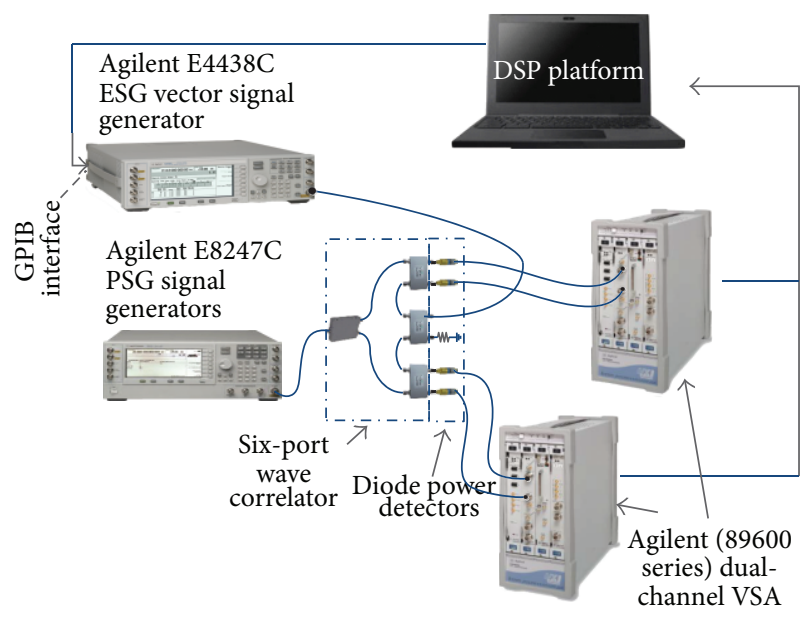

FIGURE 4: Test bench setup for the SPR Front-End.

are passed into the SPR and the voltages generated by the diode detectors are captured and digitized a four channeled mixed signal oscilloscope (infiniium MSO9404A from Agilent Technologies). The captured voltages are imported to MATLAB and using (5) the transmitted IQ data is recovered. The estimated IQ data is hence transmitted to the UE section of the MATLAB Simulink model as depicted in Figure 3 for further demodulation process to recover the transmitted bit.

\section{Measurement Results}

The performance of the communication system with an SPR front end is evaluated using the bit error rate (BER) of the communication system. The test bench was set up as described in Section 4. The RF input power to the SPR FE was $7 \mathrm{dBm}$ while the LO power was set to $10 \mathrm{dBm}$. 15362 048 IQ data points were generated from the WCDMA endto-end physical layer MATLAB model and sent at an RF frequency of $2.5 \mathrm{GHz}$ with the signal generator. 2500 samples of the generated IQ data were used in calibrating the SPR and the estimated calibration constants were used to recover the remaining IQ data points from the RF signal. To enable a plot of the BER characteristics of the receiver, the signal-to-noise ratio of the generated $I Q$ data point was varied between $-15 \mathrm{~dB}$ and $5 \mathrm{~dB}$ in steps of $2.5 \mathrm{~dB}$. Measurements were taken for the following scenarios and the corresponding references in brackets are used in the BER plot legend:

(i) complete MATLAB simulation without a receiver FE and without multipath channel fading (Matlab_wo_multipath),

(ii) complete MATLAB simulation without a receiver FE with a multipath fading channel (Matlab_w_multipath),

(iii) SPR homodyne receiver FE simulation with a multipath fading channel with memory polynomial calibration technique (SPRmp_w_multipath) using (5),

(iv) SPR homodyne receiver FE simulation with a multipath fading channel with linear combination estimation technique (SPRc_w_multipath) using (2), (4a), and $(4 b)$.

The first two cases are complete MATLAB simulations, which skip the homodyne receiver block. It assumes a perfect receiver $\mathrm{FE}$ which does not introduce any distortion or noise to the received signal. In the third and fourth case, the implemented six-port receiver front-end of Figure 4 is used and along with the signal generation and demodulation in MATLAB. Table 2 shows the measured BER and the estimated EVM of the receiver at different SNRs after the multipath fading equalization. The equalization is carried out in the WCDMA UE Rx Antenna subsystem of the MATLAB model. Figure 5 shows a plot of the BER characteristics of the communication system. The receiver calibration and IQ demodulation was done at a nonlinearity order of 2 memory depth of 4 .

From these results, it can be concluded that, in the case of a WCDMA communication system including a multipath fading channel, both calibration techniques (in the third and fourth cases) are able to provide similar BER performance to an ideal receiver with a multipath channel. Therefore, both these calibrations are able to compensate for the receiver imperfections to acceptable levels. It is worth mentioning that the calibration technique based on memory polynomials has more coefficients and therefore is more complex to implement. 


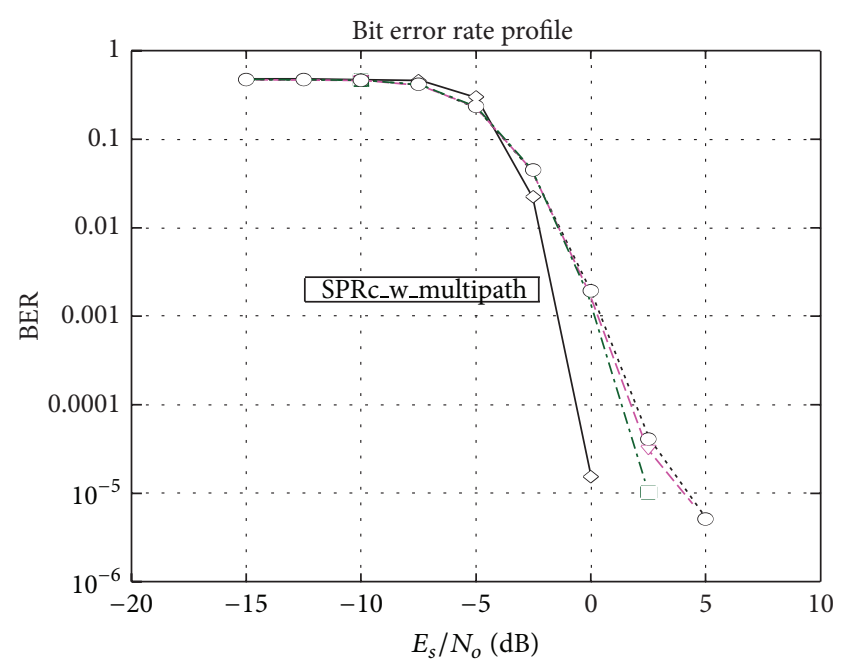

$\diamond$ Matlab_wo_multipath $\quad-\nabla-$ SPRmp_w_multipath -- - Matlab_w_multipath $\quad$.. 0. SPRc_w_multipath

FIGURE 5: BER plot of the communication system.

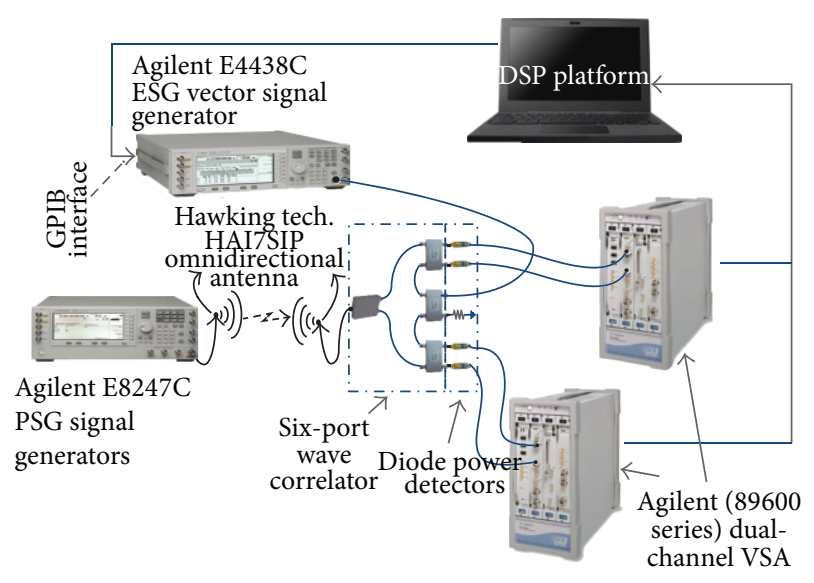

FIGURE 6: Test bench setup for the SPR Front-End using transmitting and receiving antennas.

4.1. Supplementary Result. Further hardware test was carried out using the schematic setup in Figure 6 to verify the suitability of the SPR for real communication signals with high data throughput. An LTE signal with $3 \mathrm{MHz}$ bandwidth was sent and received at $2.4 \mathrm{GHz}$ using two commercially available omnidirectional antennas (Hawking Tech. HAI7SIP). The resulting EVM of the received $I / Q$ data as compared to the transmitted data was $2 \%$. This further shows the suitability of the SPR for high data rate communication systems coupled with being multistandard and highly reconfigurable.

\section{Conclusion}

This paper investigates the viability of an SPR front end in a WCDMA communication system with a multipath fading channel. The BER profile for the communication system is plotted for four different cases. In the first case, the system is without a multipath channel effect and assumes a perfect receiver FE. In the second case, the system has a multipath channel effect and also assumes a perfect receiver FE. In the third and fourth cases, a multipath channel is considered along with an implemented SPR FE. In the third case, modified memory polynomial calibration technique is used, while in the fourth case, a less complex calibration technique using linear estimation is adopted. A comparison between the four BER plots concludes that both calibration techniques are capable of providing very good BER performances, similar to the BER of an ideal receiver with multipath channel fading. Therefore, this work concludes that it is sufficient to use the least complex calibration technique in the case of a WCDMA communication system in a multipath fading environment.

\section{Conflict of Interests}

The authors declare that there is no conflict of interests regarding the publication of this paper.

\section{References}

[1] G. Hueber, R. Stuhlberger, and A. Springer, "An adaptive digital front-end for multimode wireless receivers," IEEE Transactions on Circuits and Systems II, vol. 55, no. 4, pp. 349-353, 2008.

[2] H. Xie, O. Oliaei, P. Rakers et al., "Single-Chip Multiband EGPRS and SAW-less LTE WCDMA CMOS receiver with diversity," IEEE Transactions on Microwave Theory and Techniques, vol. 60, no. 5, pp. 1390-1396, 2012.

[3] R. Barrak, A. Ghazel, and F. Ghannouchi, "Optimized multistandard rf subsampling receiver architecture," IEEE Transactions on Wireless Communications, vol. 8, no. 6, pp. 2901-2909, 2009.

[4] J. Perez-Duenas, J. G. Wanguemert-Perez, and I. MolinaFernandez, "Novel modulation scheme and six-port based RAKE receiver for DSUWB," IEEE Transactions on Wireless Communications, vol. 8, no. 7, pp. 3628-3633, 2009.

[5] A. O. Olopade, A. Hasan, and M. Helaoui, "Concurrent dual band six port receiver for multi-standard and software defined radio applications," IEEE Transactions on Microwave Theory and Techniques, vol. 61, no. 12, pp. 4252-4261, 2013.

[6] J. Li, R. G. Bosisio, and K. Wu, "Dual-tone calibration of sixport junction and its application to the six-port direct digital millimetric receiver," IEEE Transactions on Microwave Theory and Techniques, vol. 44, no. 1, pp. 93-99, 1996.

[7] T. Hentschel, "A simple IQ-regeneration technique for six-port communication receivers," in Proceedings of the 1st International Symposium on Control, Communications and Signal Processing (ISCCSP '04), pp. 311-314, March 2004.

[8] A. Hasan and M. Helaoui, "Novel modeling and calibration approach for multiport receivers mitigating system imperfections and hardware impairments," IEEE Transactions on Microwave Theory and Techniques, vol. 60, no. 8, pp. 2644-2653, 2012.

[9] B. Laemmle, G. Vinci, L. Maurer, R. Weigel, and A. Koelpin, "An integrated 77-GHz six-port receiver front-end for angleof-arrival detection," in Proceedings of the 25th IEEE Bipolar/BiCMOS Technology and Circuits Meeting (BCTM '11), pp. 219-222, October 2011.

[10] A. Khy and B. Huyart, "A $94 \mathrm{GHz}$ radar using a six-port reflectometer as a phase/frequency discriminator," in Proceedings of 
the 2nd European Radar Conference (EURAD '05), pp. 233-236, October 2005.

[11] P. Håkansson and S. Gong, "Ultra-wideband six-port transmitter and receiver pair 3.1-4.8 GHz," in Proceedings of the Asia Pacific Microwave Conference (APMC '08), pp. 1-4, December 2008.

[12] T. Bugo, B. Klippenstein, M. Saizew, M. Woods, and M. Helaoui, "Dual-band receiver using passive six-port down-conversion technique suitable for multi-standards and SDR applications," in Proceedings of the Asia Pacific Microwave Conference (APMC '10), pp. 1312-1315, December 2010.

[13] G. Colef, P. R. Karmel, and M. Ettenberg, "New in-situ calibration of diode detectors used in six-port network analyzers," IEEE Transactions on Instrumentation and Measurement, vol. 39, no. 1, pp. 201-204, 1990.

[14] S. Lindner, F. Barbon, G. Vinci, R. Weigel, and A. Koelpin, "Initial calibration procedure of a six-port receiver system for complex data reception," in Proceedings of the 2nd European Radar Conference (EURAD '12), pp. 570-573, 2012.

[15] X. Z. Xiong and C. Iiao, "Calibration methods of microwave diode detectors," in Proceedings of the Asia-Pacific Microwave Conference (APMC '05), vol. 5, December 2005.

[16] WCDMA End-to-End Physical Layer, http://www.mathworks .com/help/comm/examples/wcdma-end-to-end-physical-layer .html. 

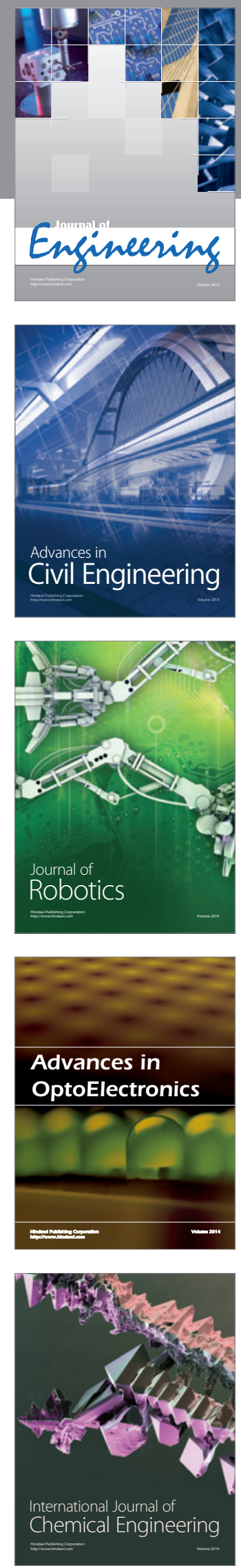

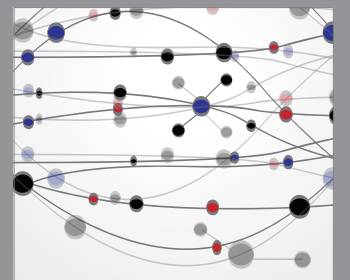

The Scientific World Journal
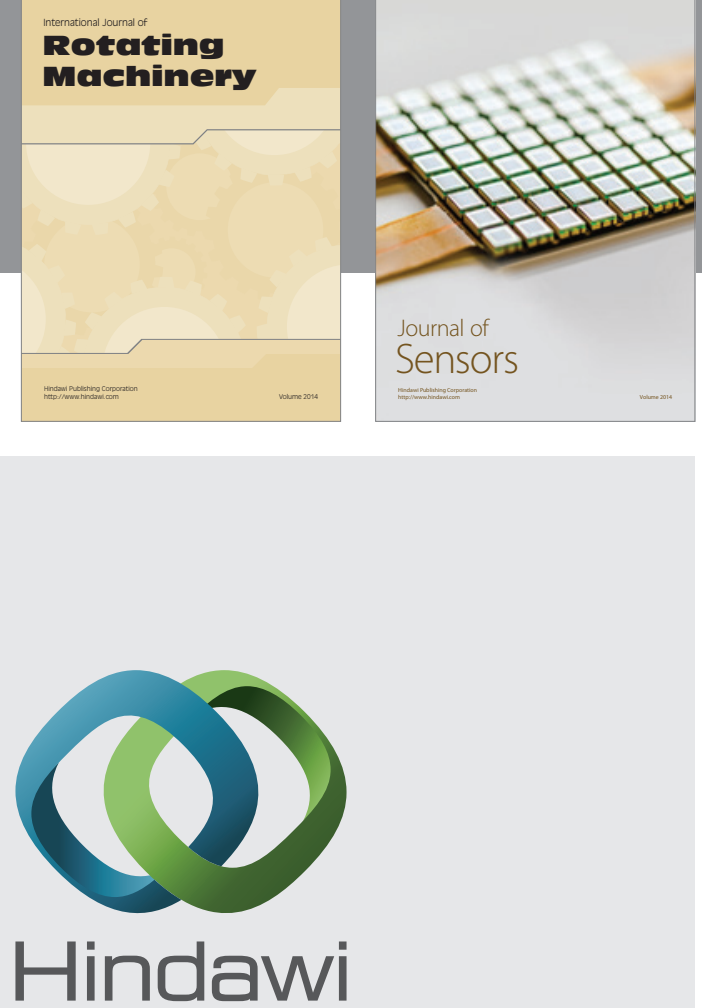

Submit your manuscripts at http://www.hindawi.com
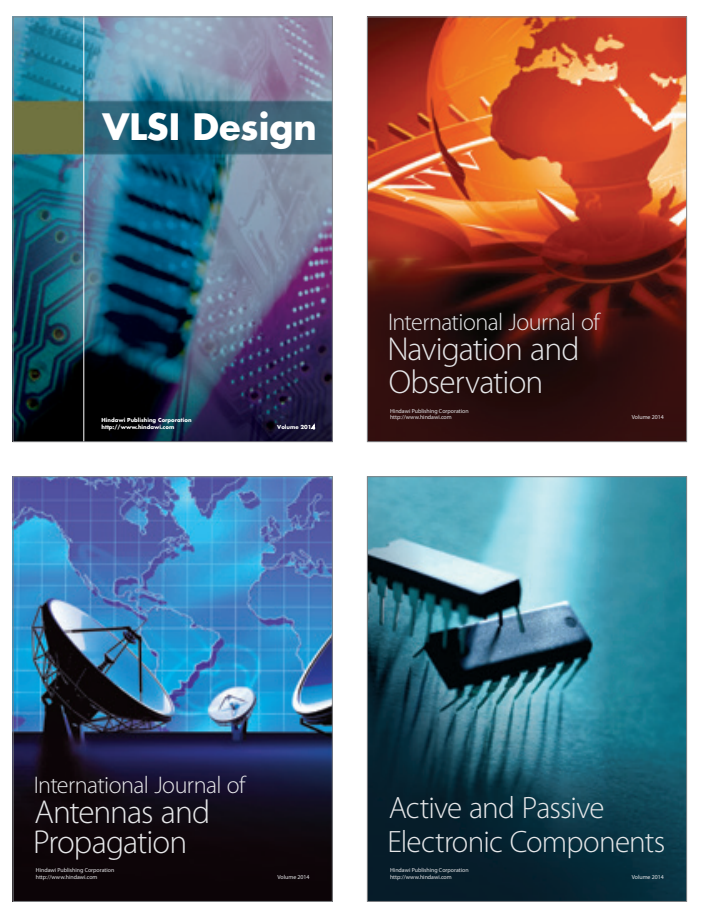
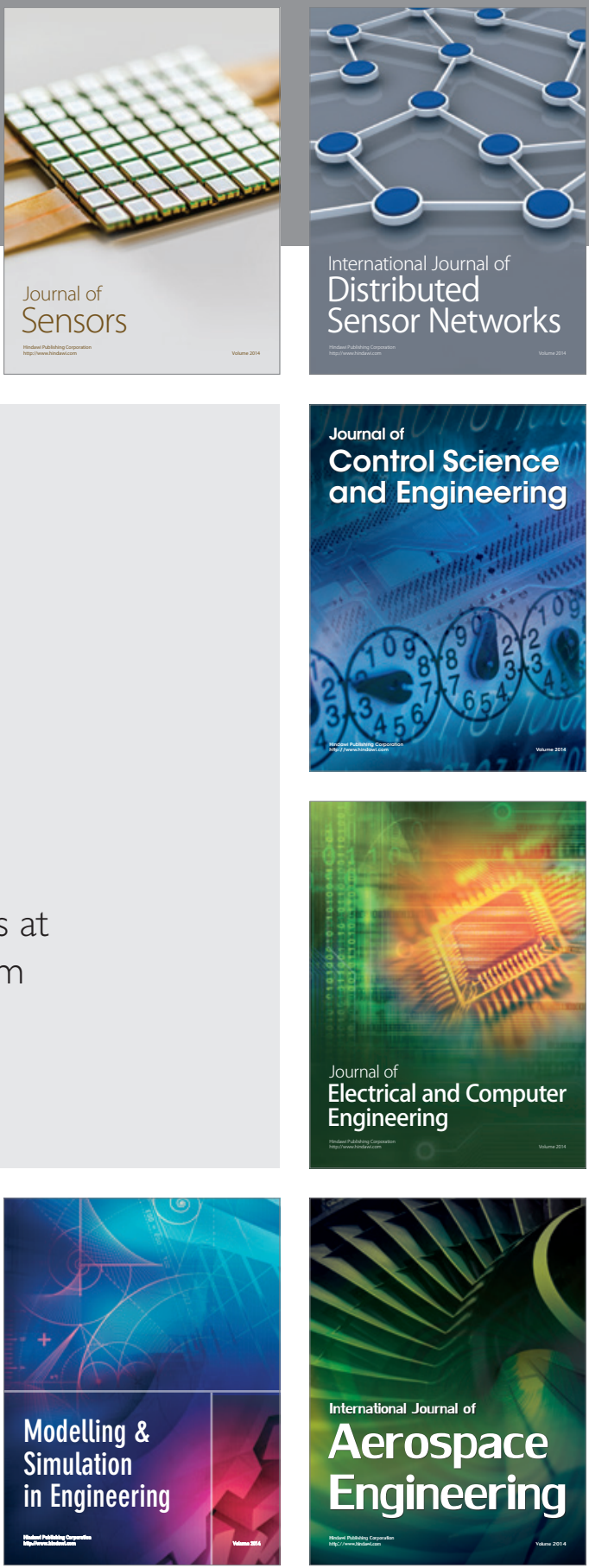

Journal of

Control Science

and Engineering
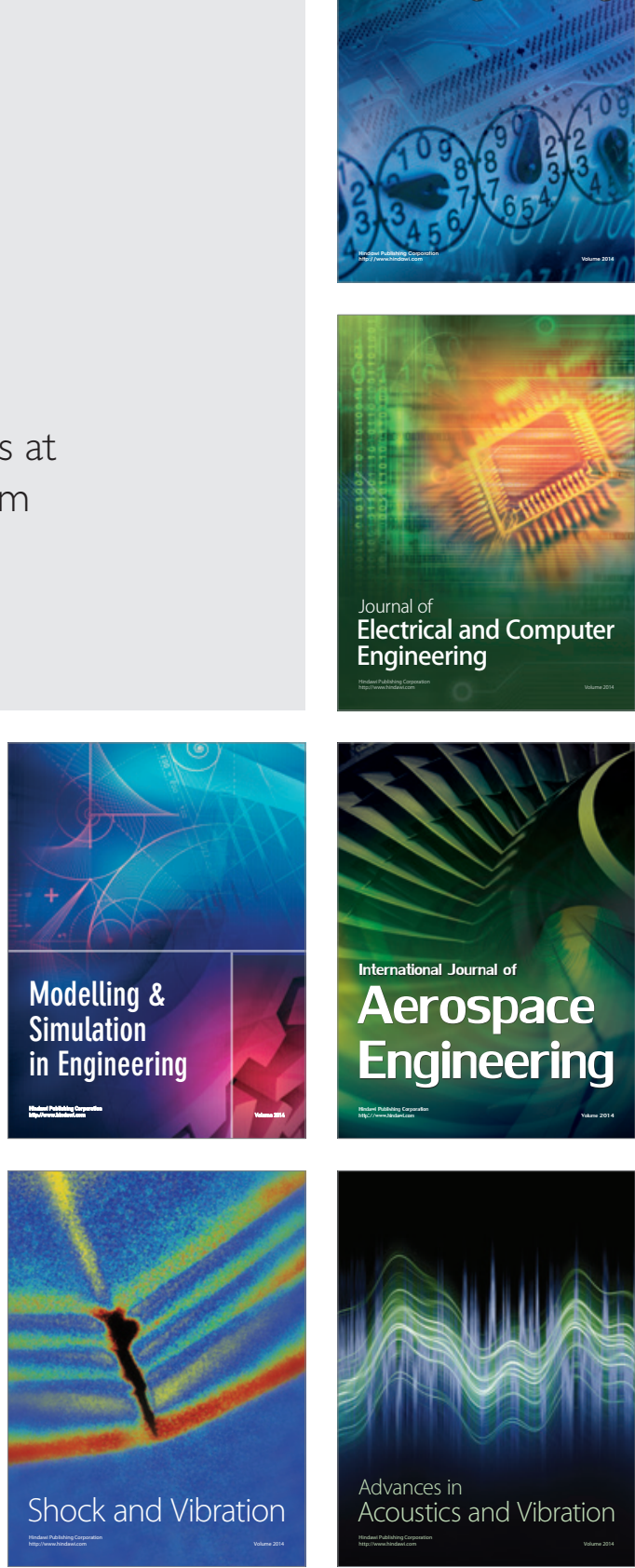